\title{
Landscape of Infantile Hemangioma Management Trends on the 100 Top-Cited Articles Since 2010: Bibliometric Analysis
}

\author{
Meng-Ju Li \\ National Taiwan University Hsin-Chu Hospital \\ Lin-Yen Wang \\ Chi-Mei Medical Center \\ Tsair-wei Chien \\ Chi-Mei Medical Center \\ Feng-Jie Lai \\ Chi Mei Medical Center \\ Kuang-Wen Liao \\ National Yang Ming Chiao Tung University \\ An-Kuo Chou ( $\nabla$ dtped124@gmail.com ) \\ National Taiwan University Hsin-Chu Hospital
}

\section{Research Article}

Keywords: infantile hemangioma, citation analysis, correlation coefficient, Social network analysis, cluster analysis

Posted Date: October 29th, 2021

DOI: https://doi.org/10.21203/rs.3.rs-1018468/v1

License: (c) (1) This work is licensed under a Creative Commons Attribution 4.0 International License. Read Full License 


\section{Landscape of Infantile Hemangioma Management Trends on}

\section{the 100 Top-Cited Articles Since 2010: Bibliometric Analysis}

Meng-Ju Li, MD ${ }^{1,2}$, Lin-Yen Wang, MD ${ }^{3,4,5}$, Tsair-Wei Chien, MBA ${ }^{6}$, Feng-Jie Lai $\mathrm{MD} P \mathrm{Ph},{ }^{7,8}$, Kuang-Wen Liao, $\mathrm{MD}^{2}$, An-Kuo Chou, $\mathrm{MD}^{1 *}$

${ }^{1}$ Department of Pediatrics, National Taiwan University Hsin-Chu Hospital, Hsinchu, Taiwan

${ }^{2}$ Department of Biological Science and Technology, National Yang Ming Chiao Tung University, Hsinchu, Taiwan

${ }^{3}$ Department of Pediatrics, Chi-Mei Medical Center, Tainan, Taiwan;

${ }^{4}$ Department of Childhood Education and Nursery, Chia Nan University of Pharmacy and Science, Tainan, Taiwan

${ }^{5}$ School of Medicine, College of Medicine, Kaohsiung Medical University, Kaohsiung, Taiwan

${ }^{6}$ Medical Research Department, Chi-Mei Medical Center, Tainan, Taiwan

${ }^{7}$ Department of Dermatology, Chi Mei Medical Center, Tainan, Taiwan

${ }^{8}$ Center for General Education, Southern Taiwan University of Science and Technology, Tainan, Taiwan

* Correspondence: dtped124@gmail.com

Short title: Infantile Hemangioma Management Trends on the 100 Top-Cited Articles

Meng-Ju Li(MJ): deary0308@gmail.com

Lin-Yen Wang (LY): yen3546@yahoo.com.tw

Tsair-Wei Chien (TW):smile@mail.chimei.org.tw

Feng-Jie Lai (FJ): lai.fengjie@gmail.com

Kuang-Wen Liao (KW): liaonms@g2.nctu.edu.tw

An-Kuo Chou (AK): dtped124@gmail.com 


\begin{abstract}
Background: An infantile hemangioma (IH) is a type of benign vascular tumor that affects babies. They usually begin during the first four weeks of life, grow until about five months of life, and then shrink in size and disappear over the next few years. Complications may include pain, bleeding, ulcer formation, heart failure, or disfigurement. Numerous articles on IH have been published in literature, sharing expanded management strategies. Nevertheless, definite treatment guideline for IH is inconclusive due to differences in heterogenous presentations, varied severity and treatment strategy among countries. This study aimed to apply the medical subject headings (MeSH terms) to predict the number of article citations using 100 top-cited IH articles (T100IHs) and dissect their characteristics including the influential authors and affiliated counties since 2010 .
\end{abstract}

Methods: By searching the PubMed Central ${ }^{\circledR}(\mathrm{PMC})$ database, the T100IHs since 2010 were downloaded. Citation analysis was performed to compare the dominant countries and authors using social network analysis (SNA) and visual representations. MeSH terms were clustered by SNA when referring their citations in articles. The prediction effect was assessed based on its correlation coefficients (CC) between impact factors(IF) of MeSH terms and the T100IHs. 
Results: We observed that (1) the top three productive countries and journals in T100IHs were the US (49\%), China (10\%), France (8\%); and Pediatrics (10\%), Br J Dermatol (6\%), Pediatr Dermatol (4\%); (2) the most cited article (PMID=25693013 with 145 citations) was authored by Christine Léauté-Labrèze from France in 2015; (3) the most cited MeSH term was pharmacology with IF equal to 42.46 in T100IHs. MeSH terms were evident in prediction power on the number of article citations (correlation coefficient $=0.46 ; \mathrm{t}=5.11 ; \mathrm{p}<0.01, \mathrm{n}=100$ ).

Conclusion: The breakthrough was made by making the citation prediction on T100IHs. MeSH terms are evident in predicting article citations using SNA and IF calculation approaches that can be applied to future research, and is not limited to the IH topic as we did in this study. .

Keywords: infantile hemangioma; citation analysis; correlation coefficient; Social network analysis; cluster analysis

\section{Background}

Infantile hemangiomas (IHs) is the most common benign tumor of infancy with occurrence rate of 5\%. Most IHs were small and innocuous, which were supposedly to be resolved spontaneously and no aggressive treatment was required. However, some of them are troublesome because of their size or location. Large IH may cause 
hemodynamic unstable, making heart failure; IH located at specific sites may cause disfigurement (e.g., facial IH), functional impairment (e.g., airway or periorbital IHs) and ulceration (e.g., at skin folds). To treat those potentially problematic, IH is essential for retaining function and even saving life in severe cases.

As Léauté-Labrèze and colleagues introduced successful experience of propranolol in treating severe and disfiguring IHs in 2008, difficult cases with hemodynamic compromised and no response to traditional management seem reach a proceeding progress[1]. This prescribed dose of propranolol was $2-3 \mathrm{mg} / \mathrm{kg} /$ day. For these critical cases, there is no debate to prescribe drug or perform invasive intervention, such as transarterial embolization[2]. However, for those mild form IHs, to treat or not may be considerable since propranolol causes adverse effects, including sleep disturbances, bronchial irritation, and clinically symptomatic bradycardia and hypotension]3].

Bibliometric analysis, focusing on a certain topic, provides insights into the contribution of particular countries and institutes, making researchers to acquire knowledge about trends of treatment[4-7]. Meanwhile, citation counts usually indicate the interest of researchers in using articles referred to their own studies[8]. Accordingly, bibliometric analysis can be used to demonstrate study hotspots and future trends in a specific disease or scientific field $[9,10]$.

Although citation analysis has been applied to identify landmark literature in 
many medical fields, such as pemphigus vulgaris[8], neurosurgery[10,11],

cardiovascular disease, urology[12], and immune-related inflammatory diseases[13],

no bibliometric analysis focusing on the topic of IHs has been published. As such, we are motivated to analyze 100 top-cited articles on IHs(T100IHs for short) and aimed to (1) investigate treatment trend through analyzing characteristics of influential authors and affiliated counties on T100IHs, (2) visualize dominant entities with dynamic Google Maps dashboards, and (3) inspect whether medical subject headings (MeSH terms) can be used to predict article citations.

\section{Methods}

\subsection{Data Sources}

As of September 3, 2021, we applied Microsoft Excel's Visual Basic for Applications (VBA) modules to extract the abstracts on the topic of infantile hemangioma (IH) since 2010. The search terms included "infantile hemangioma, treatment" in abstracts. All those top-100 cited articles (T100IHs for short) in the 2,345 downloaded abstracts were involved within 2010 and 2019 [14,15].

Only those articles labeled as journal articles were included. Others, irrelevant to the journal types of reviews and journal articles were excluded. Article citations were matched to the T100IHs for comparing entity research achievements and impact 
factors (IFs) based on MeSH terms in T100IHs.

\subsection{Data Arrangement to Fit the SNA Requirement}

We applied social network analysis (SNA) $[16,17]$ based on the Pajek software[18] to cluster primary entities. The author-made Microsoft Excel's VBA routines were used to perform SNA and draw dashboards on Google Maps.

\subsubsection{Bubbles Sized by Centrality Degree}

The number of connections for a specific MeSH term in an article were computed. The equal weight (Wi) shown in Eq. 1 was used to calculate the weighted centrality degree (CD), where L denotes the number of MeSH terms in an article, and $\mathrm{j}$ represents the location in an article byline. In SNA, each MeSH term defined as a note earns the CD Eq. 2, where $\mathrm{n}$ denotes the sample size, and the $\mathrm{CD}$ for a given MeSH term is determined by using the summed weights in all articles.

\subsubsection{All Weights Equal Article Number}

In tradition, the $\mathrm{CD}$ is primarily denoted by the number of co-occurred entities in an article. The sum of CD across all entities in all articles might not be equal the number of article in study data. As such, a particular adjustment is required to make all WCD in Eq. 2 equal the number of articles vis Eq. 1 to 3

$$
W_{i}=\frac{1}{L},(1) .
$$




$$
\begin{gathered}
\text { WCD in an article }=\left(\sum_{i=1}^{L-1} \sum_{j=i+1}^{L}\left(W_{i}+W_{j}\right)\right) \div(L-1) \\
=2 \times W_{i} \times \frac{L \times(L-1)}{2} \div(L-1) \\
=2 \times \frac{1}{L} \times \frac{L \times(L-1)}{2} \div(L-1)=1,(2)
\end{gathered}
$$

Where L is the number of co-occurred entities (e.g., authors or MeSH terms) in an article byline. Wi is the equal weight for an entity in an article byline; see Eq. 1. WCD is thus the weighted CD. In Eq. 2, Wi equals Wj based on Eq. 1. The sum of WCD for all entities in an article equals 1.0. The total WCD is equal to the number of articles, different from the traditional SNA ignoring the WCD equal to article number.

For instance, there are two MeSH terms $\mathrm{A}$ and $\mathrm{B}$ in an article. The $\mathrm{WCD}=2 \times \frac{1}{2} \times$ $\frac{2 \times(2-1)}{2} \div(2-1)=1$. Similarly, WCD equals 1 if $\mathrm{L}$ is greater than 2 . The WCD for a specific MeSH term(or other entity) $\mathrm{k}$ is the summation of Wi in all articles in Eq. 3.

$$
W C D_{k}=\sum_{j=1}^{n} W_{j},(3)
$$

Whereas $n=2,345$ in this study. The $\mathrm{WCD}_{\mathrm{k}}$ is thus sized by a bubble in SNA.

\subsubsection{All Citation Weights Equal Article Number Multiplied by Citations}

If article citations(denoted by the symbol IF) were taken into account, the weights of a specific entity(e.g., WCD in Eq. 2) is combined with IF to compute the IFWCD $_{\mathrm{k}}$ via Eqs. 4 and 5.

$$
\begin{gathered}
\text { IFWCD in an article }=I F \times\left(\sum_{i=1}^{L-1} \sum_{L=i+1}^{L}\left(W_{i}+W_{L}\right)\right) \div(L-1) \\
=I F \times 2 \times W_{i} \times \frac{L \times(L-1)}{2} \div(L-1)
\end{gathered}
$$




$$
\begin{aligned}
& =\mathrm{IF} \times 2 \times \frac{1}{L} \times \frac{L \times(L-1)}{2} \div(L-1)=I F,(4) \\
& I F W C D_{k}=\left(\sum_{j=1}^{n} \operatorname{IFWCD}_{j}\right) \div W C D_{k},(5) \\
& \text { Citations }=\sum_{k=1}^{n} I F W C D_{k},(6)
\end{aligned}
$$

Where the $I F W C D_{k}$ is the mean IF of the entity $\mathrm{k}$. All citations in articles are composed by individual $I F W C D_{k}$ in Eq. 6. Accordingly, the bubble can be sized by the $I F W C D_{k}$ for each entity.

It is worth noting that the computation of IFWCD for individual authors in articles is based on an author-weighted scheme (AWS)[17,19] instead of the equal credit in Eq. 1. As such, the first author earns the most credit (about 63\%), followed by others(e.g., the corresponding author (assumed as the last author) with about $12 \%$ credit, and other middle authors obtain the remaining portions in descending order).

\subsection{Data Presentations Using Tables and Figures}

\subsubsection{Two Tables on Affiliated Countries and Journals}

The publications of T100IHs were tabulated by country and journals over years. Impact factor $(\mathrm{IF}=$ citations/publications $)$ was calculated for each country and journal.

\subsubsection{Two Figures for Displaying Productive and Influential Countries}

Based on the 2,345 articles, the weighted publications based on AWS were computed for countries and shown on a choropleth map[20]. The x-indexes were compared in countries using the Kano diagram[22], where the publications and citations are based on the $\mathrm{x}$-core articles on $\mathrm{x}$ and $\mathrm{y}$ axis, respectively[21]. Three parts 
exist in the Kano diagram, consisting of (1) the citation-oriented at the top, (2) the unidimensional area in the middle, and (3) the productivity-oriented at the bottom.

\subsubsection{Two Figures for Authors and MeSH Terms Using SNA}

The $\mathrm{x}$-indexes of top 100 authors were dispersed on the Kano diagram[22]. The MeSH terms and journals in T100IHs were separated and clustered using SNA in Eqs. 4 and 5. The influential entities denoted by IFs were thus highlighted on a dashboard.

\subsubsection{The Prediction of Article Citations on the Weighted IFs of MeSH Terms}

The IFs of MeSH terms were computed on equal-size proportions and citations in an article. The WCDs yielded by MeSH weights were expected to predict the original citations. The computation of the weighted MeSH citations is shown in Eq. 5.

\subsubsection{The Difference in Research Topic Between Countries}

The most productive country was compared to other countries based on the frequently occurred MeSH terms shown on the forest plot[23].

\subsection{Statistics and Tools}

The correlation coefficient (CC) was used to present the predictive effect between the WCD of MeSH terms and the number of article citations. The $\mathrm{CC}$ t-value

was denoted by the formula $\left(=\mathrm{CC} \times \sqrt{\frac{n-2}{1-C C \times C C}}\right)$. A prediction equation was formulated via the simple regression analysis performed by the MedCalc statistical software, version 9.5.0.0 (MedCalc, New York, New York, USA). The significance level was set at Type I error (0.05). The study process, data, and content are presented in Additional File 1.

\section{Results}


A total of 2,345 publications were extracted from the PubMed database since 2010. The T100IHs were listed at the link[15]. The total citation counts ranged from 18 to 136 , as of July 30,2021 , in Pubmed, with a total of 3,190 citations. The mean number of citations was 31.9 times.

\subsection{The Most Productive Countries/Journals}

The most productive countries in T100IHs were the US (49\%), China (10\%), France (8\%). Most articles were published in Pediatrics (10\%), Br J Dermatol (6\%), Pediatr Dermatol (4\%) (see Tables 1 and 2). The most cited countries and journals were Germany $(\mathrm{IF}=42.3)$ and N Engl J Med $(\mathrm{IF}=100)$.

In the total 2,345 IHs-related articles, the top three countries were the US (719), China (186), and Japan (163) (Figure 1). If US states and China's provinces are applied to compare their counterparts, the top three were Italy (123), Germany (114), and Minnesota (US) (101). Readers are invited to click on the link [25] and see the details in Figures 1.

$===$ Tables 1 and 2 inserted here $===$

$===$ Figure 1 inserted here $===$

\subsection{The Most Cited Countries}

The highest x-indexes were from France (12.49), Japan (12.33). and Germany

(11.3). Readers are invited to click on the link [26] and see the details in Figure 2. $===$ Figures 2 inserted here $===$

\subsection{The Most Cited Article and Authors}

The most cited article with 145 citations(PMID=25693013) was authored by Christine Léauté-Labrèze from France in 2015[27]. The most highlighted authors (with bigger bubble with $\mathrm{x}$-index $=9,8$ in Figure 3) were Rebecca A Stockton from the 
US, followed by C H Storch(US, x-index=9.4), and Christine Leaute-Labreze (France, $\mathrm{x}$-index $=9.27)$, linked by three blue lines in Figure 3[28].

$===$ Figure 3 inserted here $===$

\subsection{The Most Cited MeSH Terms in SNA}

When Mesh terms and journals in T100IHs were jointly clustered using Eq. (5), we found that the top one was drug therapy with $\mathrm{IF}=35.4$. The most cited MeSH term was pharmacology with IF equal to 42.46 in T100IHs.

. Two journals (i.e. Br J Dermatol and Mod Pathol with $\mathrm{IF}=49$ and 13.33, respectively) are highlighted in Figure 4. Readers are advised to click on the link [29] to see their associated MeSH terms in the identical cluster.

$===$ Figure 4 inserted here $===$

\subsection{Using MeSH Terms to Predict the Number of Article Citations in T100IHs}

After using the MeSH weights to associate with article citations based on the equal weight in article. MeSH terms are evident in the predictive effect of the number of article citations $(C C==0.46 ; \mathrm{t}=5.11 ; \mathrm{p}<0.01, \mathrm{n}=100)$ (Figure 5). The regression equation is defined as article citation $(y)=y=0.8403+1.1927 \times$ weight $(x)$ of MeSH terms $\left(\mathrm{F}=26.08, \mathrm{p}=0.002, R^{2}=0.21\right)$. The slope coefficient was statistically significant $(t=5.11 ; p<0.001)$, as shown in Figure 5.

$===$ Figure 5 inserted here $===$

\subsection{Difference in research topic between the US and other countries}

Eight topics defined by MeSH terms were extracted from Figure 4[30]. When comparing proportions of counts in events (i.e. with the MeSH term) and 
Non-events(without the MeSH term), Three is only the term of education in favor of other countries $(\mathrm{p}=0.046)$ when compared to the US. Other seven topics have equivalently equal proportions in the two groups (i.e., the US and other countries).

\subsection{Online Dashboards Shown on Google Maps}

All dashboards in the figures would appear once the QR code is clicked on the links $[25,26,28-30]$. Readers are advised to examine the details about the information for each entity.

\section{Discussions}

Bibliometric analysis explores the characteristics of previously published articles based on specific and reliable parameters, which provides physicians with important quantitative information for analyzing trends and primary research concern in certain fields[31,32]. In this study, we conducted a bibliometric analysis and identified the T100IHs, focusing on IHs and related subtopics published between 2011 and 2019 in PubMed. Our study establishes a prediction model using MeSH terms to predict the number of citations based on the 100 top-cited articles downloaded from PubMed. We found that the prediction model can provide information about which MeSH terms with higher weights (i.e., the prediction power) related to the article receiving the most citation counts. Such a model offers a way to recognize the influence of recent work and the value of the articles in the field of IHs.

\subsection{Dominant Countries and Journals on IHs}


Based on the study results, the top three productive countrie in T100IHs were the US (49\%), China (10\%), France (8\%). This is in accordance with numerous bibliometric studies, where Europe and the United States dominate the production of scientific publications[8,10-13]. However, China gradually plays an important role recently. The reason might be attributed to the substantial financial support given to research and a vast number of research institutions in those countries[9,33].

In general, the main articles were from United states and Europe. There are two opposite interpretations. One aspect is issued by implying treatment strategies that are universe around the world, but similar reports were not published. On the other hand, conservative treatment may be the strategy observed in Eastern regions. This conflict may especially exist in lesions without life-threatened or complications. Nonetheless, for treating complicated lesion, multidiscipline teamwork and facility of intensive care are warranted to achieve optimal treatment effect through comprehensive care.

We also discovered that the majority of T100IHs were published in Pediatrics (10\%), Br J Dermatol (6\%), and Pediatr Dermatol (4\%). These journals are generally the top journals in the field of IHs. This could be explained by the inherent bias and intuition that researchers tended to select articles in high-impact factor journals for citation in their research[34]. 


\subsection{The most-cited articles and recent treatments in IHs}

The effects of propranolol on IHs were discovered by chance in early 2010 . Three different pharmacological targets of propranolol on IHs were illustrated. Early effects are decreased release of nitric oxide contributing to vasoconstriction, which brightened of the hemangioma surface within 1-3 days after start of therapy. Blockage of proangiogenic signals and result in growth arrest are intermediate effects. Main character of long-term effects is tumor regression by induction of apoptosis in proliferating endothelial cells[35].

The most cited article (PMID=25693013 entitled a randomized, controlled trial of oral propranolol in infantile hemangioma) was authored by Christine Léauté-Labrèze from France in 2015[27]. It was cited 145 times.

Since Léauté-Labrèze and colleagues introduced randomized controlled trial of propranolol in treating infantile hemangiomas in 2015 , oral propranolol have been widely used for treating infantile hemangioma[27]. Furthermore, clinical practice guideline from the American Academy of Pediatrics (AAP) regarding the management of IHs was conducted in 2019, illustrating problematic IHs to be treated actively[3]. Problematic IHs included lesions which are potential for disfigurement; cause life-threatening complications; cause functional impairment; present ulceration; or patients harbored underlying abnormalities. They suggested oral propranolol to be used in cases requiring systemic therapy; topical timolol in some thin and/or superficial lesions; or surgery and/or laser treatment for residual lesions[3]. 
Notably, via bibliology analysis, complicated vascular anomalies treated by sirolimus reported by Hammill and colleagues from the US was listed in the top 3 cited articles[32]. It provides insights into application of mTOR inhibitor, Sirolimus, on the treatment of complicated life-threatening vascular lesions, which were prior resistant to multiple therapies.

\subsection{Strengths and limitations}

The strength of this study is that MeSH terms were classified into research topics(e.g., several colors in Figure 4) using SNA displaying dashboards on Google Maps, which highlighted the most dominant entities in which the IH authors were interested. Readers can manipulate the links[25,26, 28-30] independently for a better understanding of the association between the entities the authors are concerned with in this study.

In addition, applying MeSH terms to predict the number of article citations is unique and innovative to identify the most dominant research topics, which will help future academic pursuits in the field of IHs. The research approach used in this study has the potential to be applied to other topics or disciplines in the future.

Moreover, the bubbles in SNA represent the proportion of publications or citations based on Eqs. 1 to 5, which are rarely expressed in literature. It is because of bubbles 
in tradition substantially influenced by the number of co-occurred entities in SNA, making the total citations (or publications) not equal to the summation of centrality degrees.

Even if those strengths mentioned above, there are still some limitations in existence. First, we used only a single database(i.e., PubMed) to extract the T100IHs. The results of this study might be different if the articles were retrieved from other major citation databases, such as Scopus, Web of Science, and Embase.

Second, we used total citations as the research achievements as of September 3, 2021. Total citation counts were significantly associated with the age of the article. The older the articles, the more citations they may receive from citing articles. The most recently published articles are at a disadvantage on the time effect[8].

Third, citation count does not directly reflect the quality of an article, but enables a quantitative evaluation of the scientific impact in a designated field[33]. Although citation statistics have been frequently criticized, analyses of citations still provide current academic development in IHs to readers with a historical perspective on its scientific progress.

Fourth, the number of article citations might be affected by many extrinsic factors, such as journal IFs and the number of authors in article bylines. Using MeSH terms to 
predict future citation counts might have some limitations and bias. More factors

should be taken into account in the future to reach a more practical and valid

prediction.

Fifth, there was only one articles having a huge number of citations described with possible reasons in Discussions. Readers are invited to read other articles in detail on PubMed by clicking on the link[26] we collected in this study.

Finally, dashboards in Figures are shown on Google Maps[25,26, 28-30]. The use of Google Maps is not free of charge using the application programming interface (API) with a paid project key. The limitation of the dashboard is not publicly accessible if no such API was applied. The process of making dashboards with MP4 video using Microsoft Excel module is provided in Additional File 1 that helps readers apply the procedures to other topics.

\section{Conclusion}

Via bibliology analysis, articles related to His were reviewed on aspects of authors' affiliated countries and institutions, showing propranolol is the main therapeutic choice for most instances from reports of Western countries.

We suggest using similar approaches to identify the most dominant entities in article topics, authors, and research institutes using SNA and Kano diagrams. 
Researchers are encouraged to exploit the characteristics and spot bursts on other

topics in the future using bibliometric analysis, not just limited to the His as we did in this study.

\section{Abbreviations}

CC: Correlation Coefficients

CD: centrality degree

IF: Impact Factors

IH: infantile hemangioma

PMC: PubMed Central®

SNA: Social network analysis

T100IHS: 100 top-cited IH articles

VBA: Visual Basic for Applications

\section{Declarations}

\section{Ethics approval and consent to participate}

Not applicable.

All data are publicly come from Pubmed library.

\section{Consent to publish}

Not applicable.

\section{Availability of data and materials}

All data used in this study is available in Additional files.

\section{Competing interests}

The authors declare that they have no competing interests.

\section{Funding}


There are no sources of funding to be declared.

\section{Authors' Contributions}

MJ and LY provided concept and designed this study, TW and FJ interpreted the data, and AK monitored the process and the manuscript. TW and KW drafted the manuscript. All authors read the manuscript and approved the final manuscript.

\section{Acknowledgements}

We thank Enago (www.enago.tw) for the English language review of this

manuscript.

\section{Additional files}

Additional file 1: study dataset

MP4 video and T100IHS at

http://www.healthup.org.tw/article/course_mb2.asp?repno=18

\section{References}

1.Léauté-Labrèze C, Dumas de la Roque E, Hubiche T, Boralevi F, Thambo JB, Taïeb A. Propranolol for severe hemangiomas of infancy. N Engl J Med. 2008;358(24):2649-2651

2. Wildgruber M, Sadick M, Müller-Wille R, Wohlgemuth WA. Vascular tumors in infants and adolescents. Insights Imaging. 2019;10(1):30.

3. Krowchuk DP, Frieden IJ, Mancini AJ, et al. Clinical Practice Guideline for the 
Management of Infantile Hemangiomas. Pediatrics. 2019;143(1).

4. Ellegaard O, Wallin JA. The bibliometric analysis of scholarly production: How great is the impact? Scientometrics. 2015;105(3):1809-1831.

5. lakeman K. Bibliometrics in a digital age: help or hindrance. Sci Prog 2018;101:293-310.

6. Hirsch JE. An index to quantify an individual's scientific research output. Proc Natl Acad Sci U S A 2005;102:16569-72.

7. Ahmad P, Dummer P, Noorani T, Asif J. The top 50 most-cited articles published in the International Endodontic Journal. Int Endod J 2019;52:803-18.

8. Yang, T. Y., Chen, C. H., Chien, T. W., \& Lai, F. J. (2021). Predicting the number of article citations on the topic of pemphigus vulgaris with the 100 top-cited articles since 2011: A protocol for systematic review and meta-analysis. Medicine, 100(31), e26806.

9. Wang CY, Li BH, Ma LL, et al. . The top-100 highly cited original articles on drug therapy for ventilator-associated pneumonia. Front Pharmacol 2019;10:108.

10. Almutairi O, Albakr A, Al-Habib A, Ajlan A. The top-100 most-cited articles on meningioma. World Neurosurg 2017;107:1025-32. e5.

11.Nasir SAR, Gilani JA, Fatima K, Faheem U, Kazmi O, Siddiqi J, et al. . Top 100 most-cited articles on spontaneous intracerebral hemorrhage: a bibliometric 
analysis. World Neurosurg 2018;110:445-9. e6.

12. Nason GJ, Tareen F, Mortell AJCUAJ. The top 100 cited articles in urology: an update. Can Urol Assoc J 2013;7:E16.

13, Yin X, Cheng F, Wang X, Mu J, Ma C, Zhai C, et al. . Top 100 cited articles on rheumatoid arthritis: a bibliometric analysis. Medicine

(Baltimore) 2019;98:e14523.

14. Chien TW. 2345 articles extracted from Pubmed. 2021/8/3 available at https://pubmed.ncbi.nlm.nih.gov/?term=infantile+hemangioma $\% 2 \mathrm{C}+$ trea tment\&filter=years.2010-2021

15. Chien TW. T100IHs extracted from Pubmed. 2021/8/3 available at http://www.healthup.org.tw/html100/Hemangioma100.htm

16. Yie KY, Chien T W, Yeh YT, Chou W, Su S B. Using Social Network Analysis to Identify Spatiotemporal Spread Patterns of COVID-19 around the World: Online Dashboard Development. International journal of environmental research and public health 2021; 18(5): 2461.

17. Kan, W. C., Chou, W., Chien, T. W., Yeh, Y. T., \& Chou, P. H. (2020). The Most-Cited Authors Who Published Papers in JMIR mHealth and uHealth Using the Authorship-Weighted Scheme: Bibliometric Analysis. JMIR mHealth and uHealth, 8(5), e11567. 
18. Batagelj V, Mrvar A. Pajek — analysis, and visualization of large networks. In:

Jünger M, Mutzel P, editors. Graph drawing software. Berlin: Springer; 2003. pp. $77-103$.

19. Chien T, Wang H, Lai F. Applying an Author-Weighted Scheme to Identify the Most Influential Countries in Research Achievements on Skin Cancer: Observational Study. JMIR Dermatol 2019;2(1):e11015

20. Chien TW, Wang HY, Hsu CF, Kuo SC. Choropleth map legend design for visualizing the most influential areas in article citation disparities: A bibliometric study. Medicine (Baltimore). 2019 Oct;98(41):e17527.

21. Fenner T, Harris M, Levene M, Bar-Ilan J. A novel bibliometric index with a simple geometric interpretation. PLoS ONE. 2018;13(7):e0200098.

22. Kano N, Seraku N, Takahashi F, Tsuji S. Attractive quality and must-be quality. $J$ Jpn Soc Quality Control. 1984;41:39-48.

23. Chou PH, Yeh YT, Kan WC, Chien TW, Kuo SC. Using Kano diagrams to display the most cited article types, affiliated countries, authors and MeSH terms on spinal surgery in recent 12 years. Eur J Med Res. 2021 Feb 23;26(1):22.

24. Yan YH, Chien TW. The use of forest plot to identify article similarity and differences in characteristics between journals using medical subject headings terms: A protocol for bibliometric study. Medicine (Baltimore). 2021 Feb 
12;100(6):e24610. doi: 10.1097/MD.0000000000024610. PMID: 33578568;

PMCID: PMC7886407.

25. Chien TW. Figure 1 in this study. 2021/8/1 available at http://www.healthup.org.tw/gps/Hemangiomawd.htm

26. Chien TW. Figure 2 in this study. 2021/8/1 available at http://www.healthup.org.tw/gps/Hemangiomakanocty.htm

27. Léauté-Labrèze C, Hoeger P, Mazereeuw-Hautier J, Guibaud L, Baselga E, Posiunas G, Phillips RJ, Caceres H, Lopez Gutierrez JC, Ballona R, Friedlander SF, Powell J, Perek D, Metz B, Barbarot S, Maruani A, Szalai ZZ, Krol A, Boccara O, Foelster-Holst R, Febrer Bosch MI, Su J, Buckova H, Torrelo A, Cambazard F, Grantzow R, Wargon O, Wyrzykowski D, Roessler J, Bernabeu-Wittel J, Valencia AM, Przewratil P, Glick S, Pope E, Birchall N, Benjamin L, Mancini AJ, Vabres P, Souteyrand P, Frieden IJ, Berul CI, Mehta CR, Prey S, Boralevi F, Morgan CC, Heritier S, Delarue A, Voisard JJ. A randomized, controlled trial of oral propranolol in infantile hemangioma. N Engl J Med. 2015 Feb 19;372(8):735-46.

28. Chien TW. Figure 3 in this study. 2021/8/1 available at 1http://www.healthup.org.tw/gps/Hemangiomakanoaif.htm 
29. Chien TW. Figure 4 in this study. 2021/8/1 available at http://www.healthup.org.tw/gps/Hemangiomakanomesh.htm

30. Chien TW. Figure 6 in this study. 2021/8/1 available at 1http:/www.healthup.org.tw/gps/Hemangiomakanororest.htm

31. Glänzel W, Moed HF. Journal impact measures in bibliometric research. Scientometrics 2002;53:171-93.

32. Mingers J, Leydesdorff L. A review of theory and practice in scientometrics. Eur $J$ Oper Res 2015;246:01-19.

33. Pena-Cristóbal M, Diniz-Freitas M, Monteiro L, Diz Dios P, Warnakulasuriya S. The 100 most cited articles on oral cancer. J Oral Pathol Med 2018;47:33344.

34. Berlinberg A, Bilal J, Riaz IB, Kurtzman DJB. The 100 top-cited publications in psoriatic arthritis: a bibliometric analysis. Int J Dermatol 2019;58:1023-34.

35. Storch $\mathrm{CH}$, Hoeger PH. Propranolol for infantile haemangiomas: insights into the molecular mechanisms of action. Br J Dermatol. 2010;163(2):269-274.

32. Hammill AM, Wentzel M, Gupta A, et al. Sirolimus for the treatment of complicated vascular anomalies in children. Pediatr Blood Cancer. 2011;57(6):1018-1024.

33. Zhu XP, Ban Z. Citation Count Prediction Based on Academic Network Features, 
in 2018 IEEE 32nd International Conference on Advanced Information Networking and Applications (AINA); 2018. 534-541, doi:10.1109/AINA.2018.00084.

\section{Figures Legends}

Figure 1 Geographical distribution of countries for publications on the IM topic since 2010(http://www.healthup.org.tw/gps/Hemangiomawd.htm)

Figure 2 The highest research achievements for countries with $\mathrm{x}$-index

Figure 3 The highest research achievements for authors with $\mathrm{x}$-index

Figure $4 \mathrm{MeSH}$ terms with journals and their impact factors highlighted by performing cluster analysis

Figure 5 Using MeSH terms to predict the number of articles based on T100IM Figure 6 Comparison of research topics on MeSH terms between the US and other countries based on T100IM 
Figures

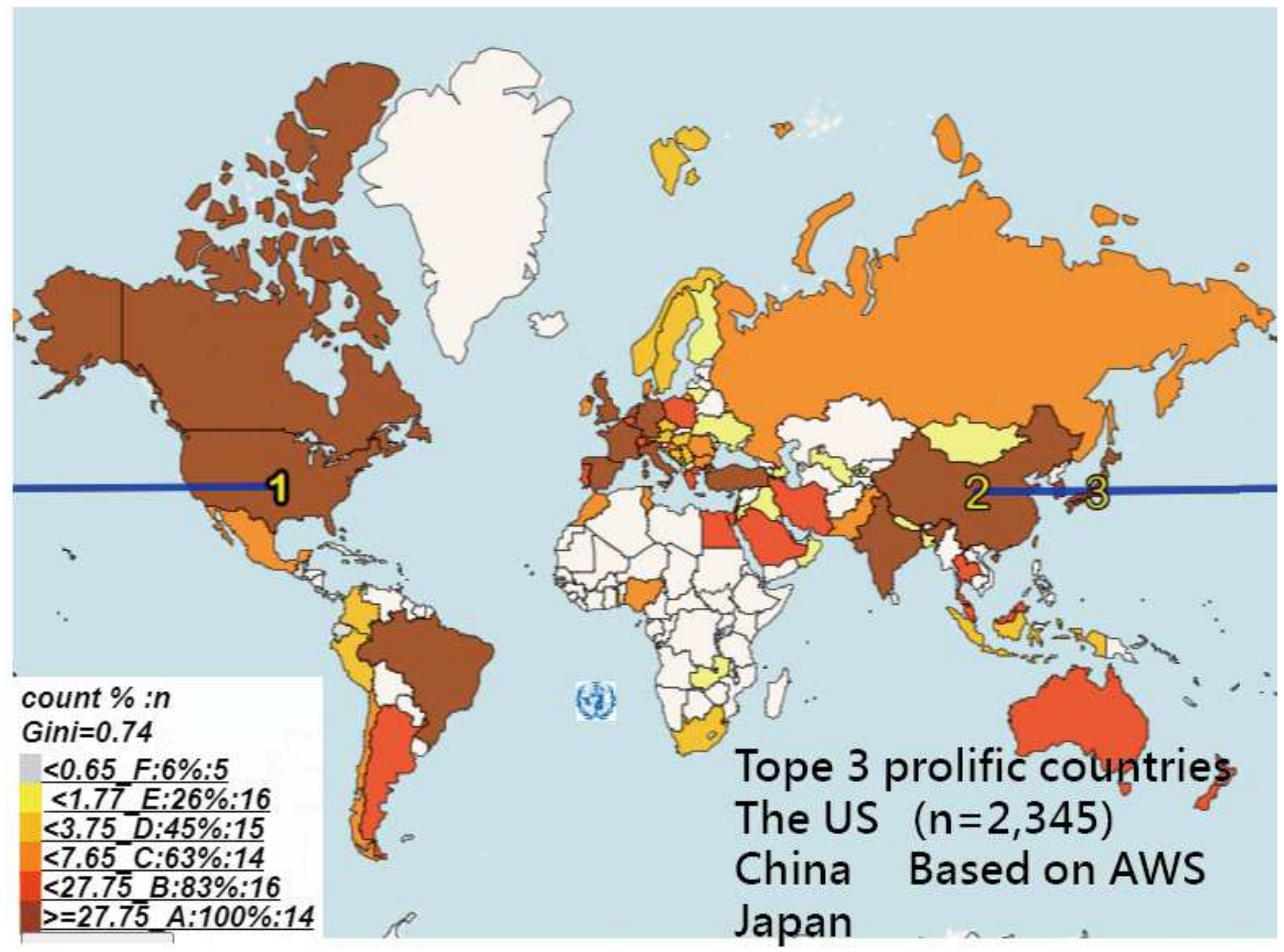

Figure 1

Geographical distribution of countries for publications on the IM topic since 2010(http://www.healthup.org.tw/gps/Hemangiomawd.htm) 


\section{Citations at x-core}

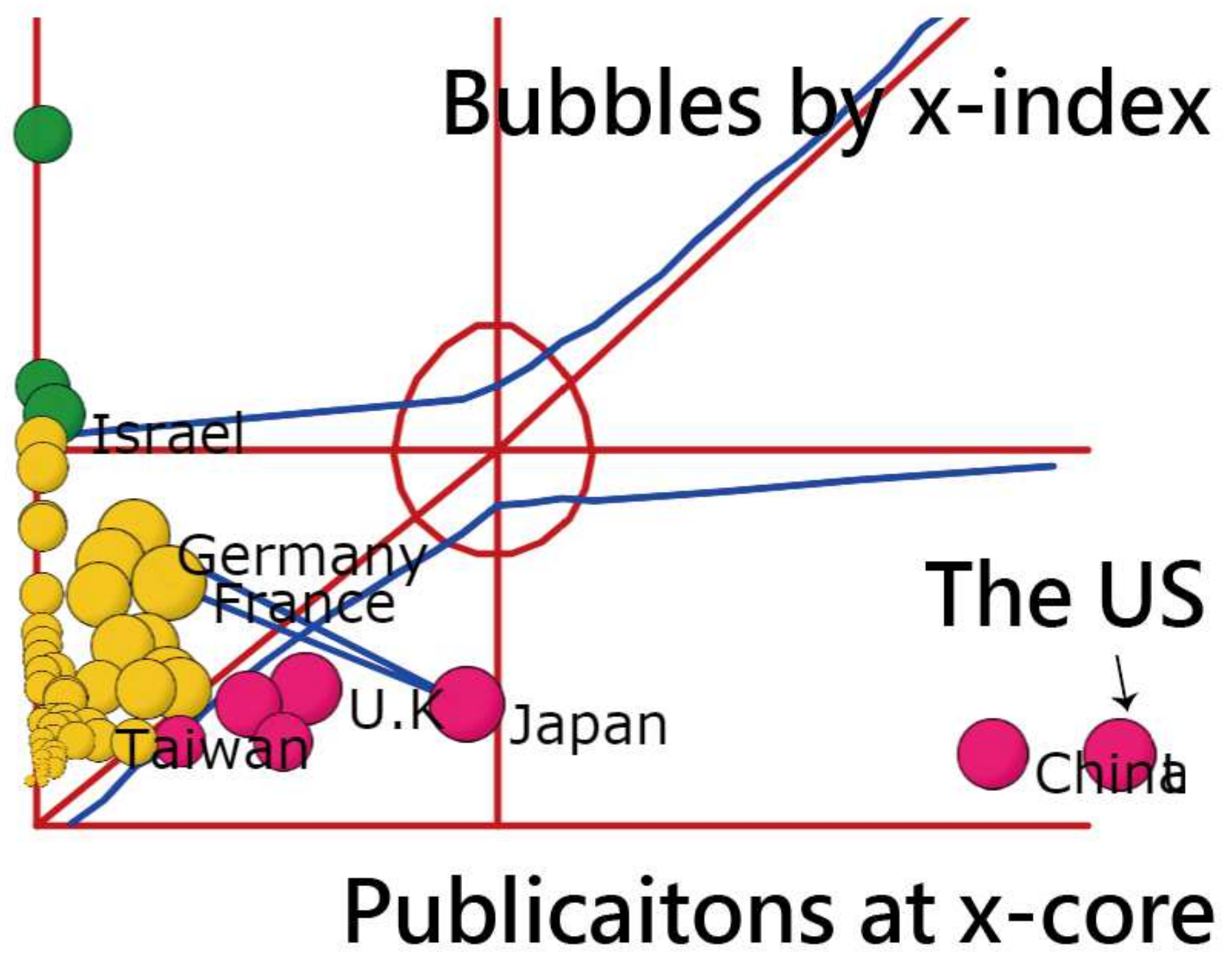

Figure 2

The highest research achievements for countries with $\mathrm{x}$-index 


\section{Citations at $\mathrm{x}$-core}

Stockton, Rebecca A

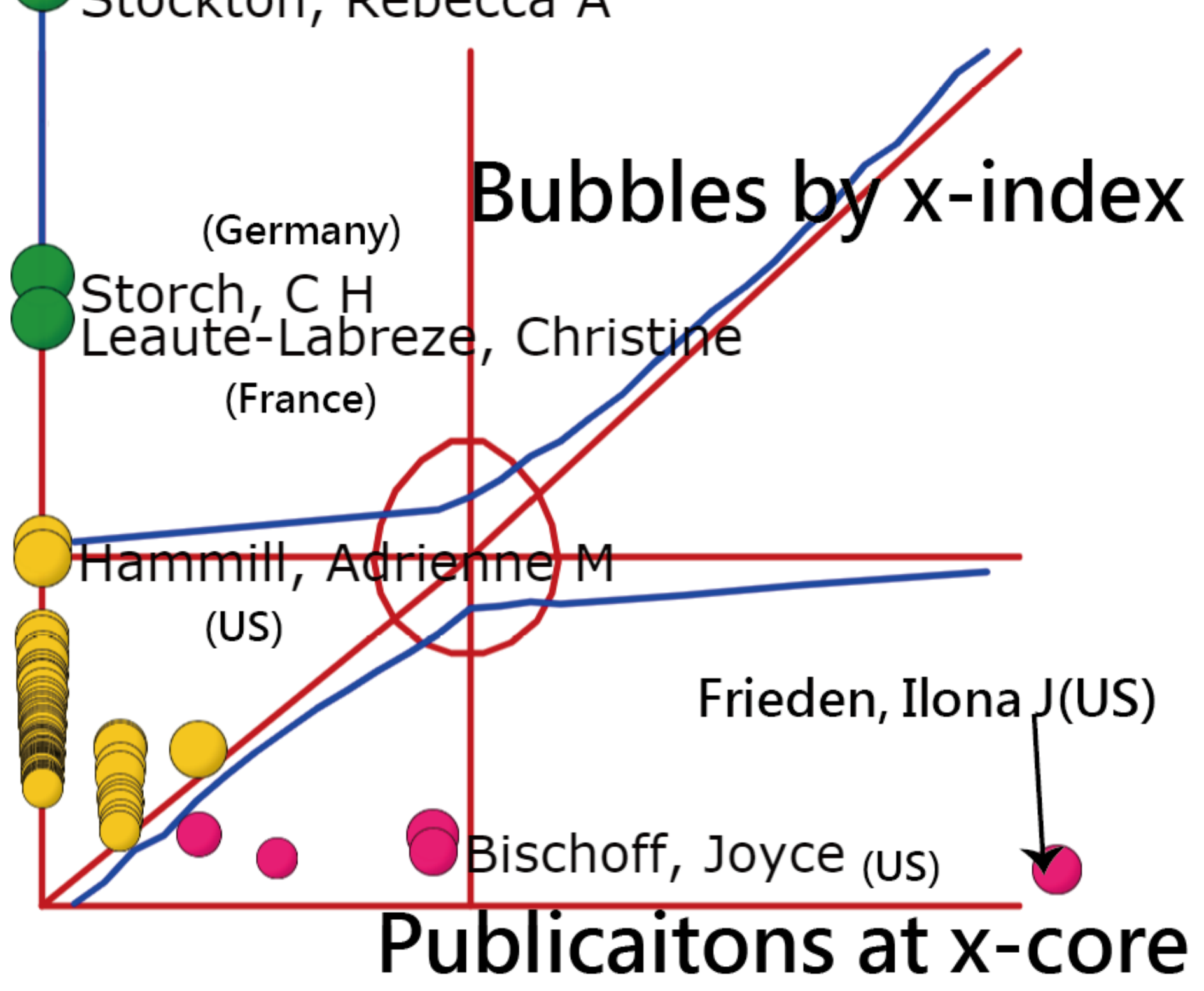

Figure 3

The highest research achievements for authors with $\mathrm{x}$-index 


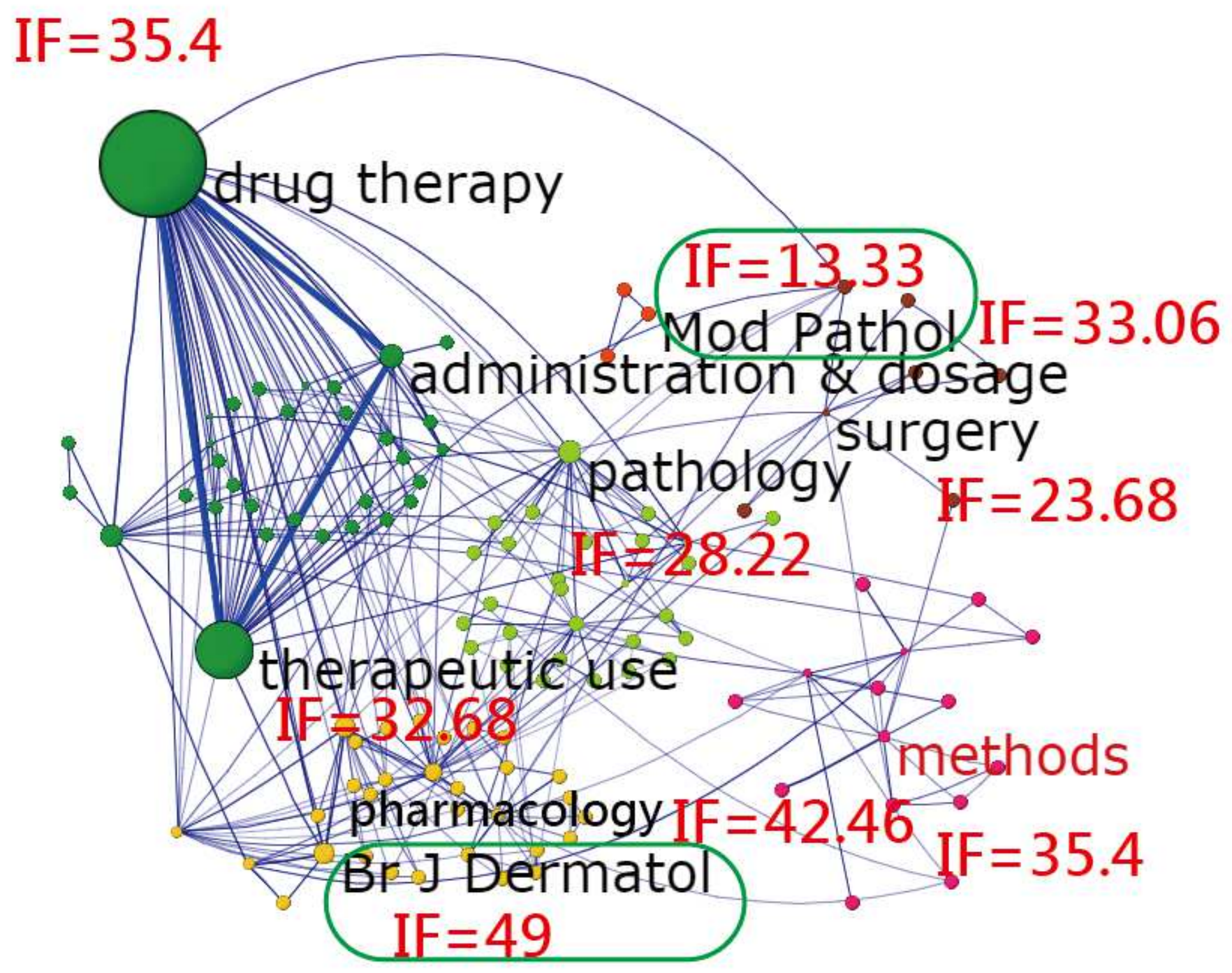

Figure 4

MeSH terms with journals and their impact factors highlighted by performing cluster analysis 


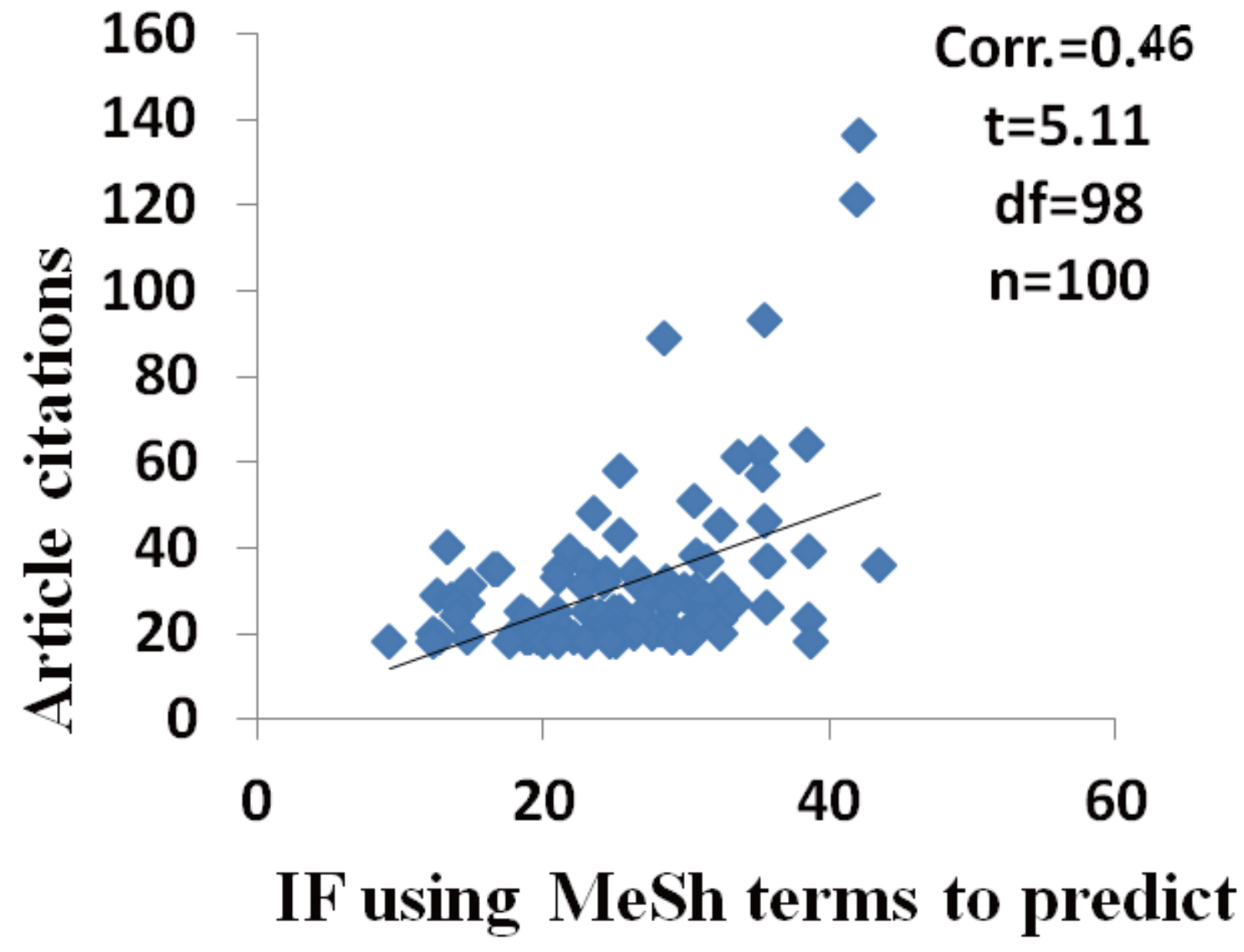

Figure 5

Using MeSH terms to predict the number of articles based on T100IM

\begin{tabular}{|c|c|c|c|c|c|c|c|c|c|c|c|c|}
\hline MeSH term & $\begin{array}{l}\text { The U } \\
\text { Events }\end{array}$ & $\begin{array}{l}\text { is ot } \\
\text { No Even }\end{array}$ & $\begin{array}{l}\text { Dthers } \\
\text { nt Eve }\end{array}$ & nts No Event & $\begin{array}{r}\text { Odds } \\
1.0\end{array}$ & & $M-H_{B}$ & Random $95 \% \mathrm{CI}$ & & p-value & weight(\%) & $n 1 / n 2$ \\
\hline standards & 19 & 30 & 29 & 22 & & & 20.480 & $(0.22,1.07)$ & -1.80 & 0.072 & 22.8 & 4951 \\
\hline communication & & 40 & 6 & 45 & & {[} & 1.688 & $(0.55,5.16)$ & 0.92 & 0.359 & 11.6 & 4951 \\
\hline methods & 2 & 47 & 3 & 48 & & & 0.681 & $(0.11,4.26)$ & -0.41 & 0.681 & 4.3 & 4951 \\
\hline clinical competence & 3 & & 3 & 48 & & & 1.043 & $(0.20,5.44)$ & 0.05 & 0.960 & 5.3 & 4951 \\
\hline educational measurem & t 19 & 30 & 29 & 22 & & & 0.480 & $(0.22,1.07)$ & -1.80 & 0.072 & 22.8 & 4951 \\
\hline psychology & 12 & 37 & 17 & 34 & & & 0.649 & $(0.27,1.55)$ & -0.97 & 0.331 & 19.1 & 4951 \\
\hline education & 2 & 47 & 9 & 42 & & & 0.199 & $(0.04,0.97)$ & -2.00 & 0.046 & 5.8 & 4951 \\
\hline curriculum & 4 & & 6 & 45 & & & 0.667 & $(0.18,2.52)$ & -0.60 & 0.550 & 8.2 & 4951 \\
\hline Overall & & & & & & & 260 & 42 089 ) & -2.55 & 0.011 & 100 & \\
\hline
\end{tabular}

$$
\text { I2 }=0.0 \mathrm{Tau}=0.0
$$

Favors Others

Favors The US

Figure 6

Comparison of research topics on MeSH terms between the US and other countries based on T100IM 


\section{Supplementary Files}

This is a list of supplementary files associated with this preprint. Click to download.

- appendix1.txt 